\title{
Markov-modulated stochastic recursive equations with applications to delay-tolerant networks
}

\author{
Dieter Fiems ${ }^{1}$
}

\begin{abstract}
We investigate Markov-modulated stochastic recursive equations. This class includes multi-type branching processes with immigration as well as linear stochastic equations. Conditions are established for the existence of a stationary solution and expressions for the first two moments of this solution are found. Furthermore, the transient characteristics of the stochastic recursion are investigated: we obtain the first two moments of the transient solution as well. Finally, to illustrate our approach, the results are applied to the performance evaluation of packet forwarding in delay-tolerant mobile ad-hoc networks.
\end{abstract}

\section{INTRODUCTION}

In this contribution, we investigate a stochastic recursive equation of a form related to both branching processes and linear stochastic equations which operates in a random environment. Both steady-state and transient behaviour are investigated and, in either case, expressions for the first two moments are established. Our results are then applied in the context of packet forwarding in delay-tolerant mobile ad-hoc networks.

The object of this paper is equations of the type

$$
X_{n+1}=A_{n}\left(X_{n}\right)+B_{n},
$$

where $X_{n} \in \mathbb{R}^{M}$ are non-negative column vectors. $B_{n} \in \mathbb{R}^{M}$, which we call the "immigration" term, is assumed to be stationary ergodic and $A_{n}$ are a sequence of independent and identically distributed (i.i.d.) stochastic processes. Moreover, the processes $A_{n}$ are assumed to have a divisibility property: if for some $k, x=x^{0}+x^{1}+\ldots+x^{k}$ then $A_{n}(x)$ can be represented as $A_{n}(x)=\sum_{i=0}^{k} \widehat{A}_{n}^{(i)}\left(x^{i}\right)$ where $\left\{\widehat{A}_{n}^{(i)}\right\}_{i=0,1,2, \ldots, k}$ are identically distributed with the same distribution as $A_{n}(\cdot)$, but they need not be independent. An example of an integervalued process $A(x)$ that is divisible is the number of arrivals at an $\mathrm{M} / \mathrm{G} / 1$ queue during the service time of $x$ customers. A non-descrete example is the time to serve all the customers that arrive at an $\mathrm{M} / \mathrm{G} / 1$ queue during some time $x$.

The above stochastic recursive equations include various types of stochastic processes: stochastic difference equations (also called stochastic auto-regressive processes), branching processes in a discrete state space and branching processes in a continuous state space. These processes have in common that the expectation of the state, $\mathrm{E}\left[X_{n}\right]$, has the same linear dynamics: there is some matrix $\mathcal{A}$ such that $\mathrm{E}\left[X_{n+1}\right]=$ $\mathcal{A} \mathrm{E}\left[X_{n}\right]+\mathrm{E}\left[B_{n}\right]$.

The main objective of this work is the introduction of a random environment $Y_{n}$ in the above class of recursive equations (which we assume for simplicity to be a function of a state of some finite state Markov chain). More precisely, we

\author{
Eitan Altman ${ }^{2}$
}

introduce equations of the type

$$
X_{n+1}=A_{n}\left(X_{n}, Y_{n}\right)+B_{n}\left(Y_{n}\right) .
$$

where $B_{n}$ is the immigration process, and $Y_{n}$ is a Markov chain that can be used to produce a correlation between $B_{n}$ and $A_{n}$ as well as to remove the independence assumption on $A_{n}$.

The theoretical foundations are then applied to various models of delay-tolerant ad-hoc networks whose dynamics can be described by the stochastic recursive equations under investigation. In particular, the impact of the random environment on the performance of these networks is investigated.

Before proceeding to our main results, we survey some related literature. A special case of our framework are branching processes with a random environment. These have been well studied, both with and without immigration; see the survey [2] and the references therein. For example, conditions are presented for the extinction when the random environment is stationary ergodic. Further, the stability, strong law of large numbers and central limit theorems for multi-type branching processes with immigration in a random environment have been studied in [9], [14].These processes find applications in very diverse fields, including biological systems and queueing theory. For example, McNamara et. Al [11] consider an asexual species with non-overlapping generations. Individuals born some a year, reach maturity and reproduce one year later and then die. The number of individuals of the different genotypes in the consecutive years constitute a multi-type branching process. A numerical example is presented for which the expected population size has the same growth rate with and without a random environment. However, the probability that the population gets extinct is different in the two cases, being equal to one in the case of the random environment.

Prime examples in queueing theory where branching processes with immigration play a major role, include infinite server queues [4], processor sharing queues [7], [12], as well as various polling systems. Resing [13] already demonstrated that the numbers of customers in the different queues of a polling system at polling instants are described by a multi-type branching process with immigration. Similarly, station times — the time the server remains with a particular queue - at polling instants are described by such a branching process as well, albeit with a continuous state space [1].

Many of the above queueing models have natural extensions that involve random environments. A polling example that can be modelled in our framework is studied in [10]. Every time one of the queues empties, some parameters can change 
at random. Another application is a polling system in which the polling order of the queues is determined according to a Markov chain: if the $n$th station being polled is station $i$, then the probability that the next one to be polled is $j$ is given by the transition probability $p_{i j}$. The infinite server queue with random environment has been studied recently in [3], [5]. These authors assume a framework of i.i.d. exponentially distributed interarrivals and i.i.d. exponentially distributed service times. Our approach allows, in contrast, to obtain explicit expressions for the first and second moments in the more general setting of general stationary ergodic arrivals and general independent bounded service time, with Markov random environment.

The theory that we develop allows us to obtain the two first moments of the stochastic recursive equations under very general assumptions on the immigration process: it is only assumed to be stationary ergodic.

The remainder of this paper is organised as follows. In the next section, the stochastic recursive equation is introduced and some notation is established. Section III is concerned with conditions for the existence of a stationary solution and expressions for the first two moments of this stationary solution. Transient characteristics are found in Section IV. With the results established, we investigate various applications of our framework in the context of delay-tolerant mobile adhoc networks in Section V. Finally conclusions are drawn in Section VI.

\section{Stochastic MOdeL}

We consider the sequence of random column vectors $X_{n} \in$ $\mathbb{R}^{M}$, adhering to the stochastic recursive equation,

$$
X_{n+1}=A_{n}\left(X_{n}, Y_{n}\right)+B_{n}\left(Y_{n}\right), \quad n \in \mathbb{Z} .
$$

Here $Y=\left\{Y_{n}\right\}$ denotes a Markov chain, taking values on a finite state-space $\Theta=\{1,2, \ldots, N\}$ whereas $A_{n}$ and $B_{n}$ denote random vector-valued functions with domain $\mathbb{R}^{M} \times \Theta$ and $\Theta$, respectively. The functions $A_{n}(\cdot, i)$ are independent random variables for all $i \in \Theta, n \in \mathbb{Z}$ and further adhere to the following assumptions.

- For each $y \in \Theta, A_{n}(\cdot, y)$ has a divisibility property. Let $x=x^{1}+x^{2}+\ldots+x^{k} \in \mathbb{R}^{M}$, then $A_{n}(x, y)$ has the following representation,

$$
A_{n}(x, y)=\sum_{i=1}^{k} \hat{A}_{n}^{(i)}\left(x^{i}, y\right),
$$

whereby $\hat{A}_{n}^{(i)}(\cdot, y), i=1, \ldots, k$, are identically distributed, but not necessarily independent, with the same distribution as $A_{n}(\cdot, y)$.

- For each $y \in \Theta, A_{n}(\cdot, y)$ is linear in the mean,

$$
\mathrm{E}\left[A_{n}(x, y)\right]=\mathcal{A}_{y}^{(n)} x, \quad x \in \mathbb{R}^{M}, y \in \Theta, n \in \mathbb{Z} .
$$

Here $\left\{\mathcal{A}_{y}^{(n)}, y \in \Theta, n \in \mathbb{Z}\right\}$ is a set of fixed $M \times M$ matrices. Further, for each $y \in \Theta$, the correlation matrix of $A_{n}(x, y)$ is linear in $x x^{\prime}$ and in $x$. For all $x=$ $\left[x^{1}, \ldots, x^{M}\right] \in \mathbb{R}^{M}$, we have the following representation,

$$
\mathrm{E}\left[A_{n}(x, y) A_{n}^{\prime}(x, y)\right]=F_{y}^{(n)}\left(x x^{\prime}\right)+\sum_{j=1}^{M} x^{j} \Gamma_{y, j}^{(n)},
$$

$y \in \Theta, n \in \mathbb{Z}$. For each $y \in \Theta$ and $n \in Z, F_{y}^{(n)}$ is a linear operator that maps $M \times M$ non-negative definite matrices on $M \times M$ non-negative definite matrices and satisfies $F_{y}^{(n)}(0)=0$. Further, $\left\{\Gamma_{y, j}^{(n)}, y, j \in \Theta, n \in \mathbb{Z}\right\}$ is a set of fixed $M \times M$ matrices.

We mention some processes $A_{n}(\cdot, y)$ for which the assumptions (2) to (4) hold. A first example constitutes the class of linear stochastic recursive equations, $X_{n+1}=A_{n+1}\left(Y_{n}\right) X_{n}+$ $B_{n}\left(Y_{n}\right)$. Here $A_{n+1}(y)$ constitutes a sequence of independent and identically distributed random matrices for all $y \in \Theta$. Multi-type branching processes are a second example, either with a continuous or a discrete state-space. Examples of a branching processes with discrete state spaces are developed in Section V. For an example of branching process with a continuous state space, the reader is referred to [1]. Finally, we note that any combination of the previous processes also complies with assumptions (2) to (4).

Before proceeding to our main results, we introduce some additional notation. Let $p_{i j}^{(n)}=\operatorname{Pr}\left[Y_{n+1}=j \mid Y_{n}=i\right]$ denote the transition probability of the Markov chain $Y_{k}$ at time $n$ $(i, j \in \Theta)$ and let $P^{(n)}=\left[p_{i j}^{(n)}\right]$ denote the corresponding transition matrix. The probability that the Markov chain is in state $k$ at slot $n$ is denoted by $\pi_{k}^{(n)}=\operatorname{Pr}\left[Y_{n}=k\right]$. For the immigration process $B_{n}$, the following notation is introduced for the first and second order moments,

$$
b_{i}^{(n)}=\mathrm{E}\left[B_{n}(i)\right], \quad \mathcal{B}_{i j}^{(m, n)}=\mathrm{E}\left[B_{m}(i) B_{n}(j)\right]
$$

Finally, the following block matrices and block vector are defined to simplify further notation,

$$
\begin{aligned}
\hat{\mathcal{A}}^{(n)}= & {\left[\begin{array}{cccc}
\mathcal{A}_{1}^{(n)} p_{11}^{(n)} & \mathcal{A}_{2}^{(n)} p_{21}^{(n)} & \ldots & \mathcal{A}_{N}^{(n)} p_{N 1}^{(n)} \\
\mathcal{A}_{1}^{(n)} p_{12}^{(n)} & \mathcal{A}_{2}^{(n)} p_{22}^{(n)} & \ldots & \mathcal{A}_{N}^{(n)} p_{N 2}^{(n)} \\
\vdots & & \ddots & \vdots \\
\mathcal{A}_{1}^{(n)} p_{1 N}^{(n)} & \mathcal{A}_{2}^{(n)} p_{2 N}^{(n)} & \ldots & \mathcal{A}_{N}^{(n)} p_{N N}^{(n)}
\end{array}\right], } \\
\hat{b}^{(n)}= & \sum_{k \in \Theta} \pi_{k}^{(n)}\left[\begin{array}{c}
p_{k 1}^{(n)} b_{k}^{(n)} \\
p_{k 2}^{(n)} b_{k}^{(n)} \\
\vdots \\
p_{k N}^{(n)} b_{k}^{(n)}
\end{array}\right],
\end{aligned}
$$

and,

$\hat{\mathcal{B}}^{(m, n)}=$

$$
\sum_{j \in \Theta} \pi_{j}^{(m)}\left[\begin{array}{cccc}
\mathcal{B}_{j 1}^{(m, n)} p_{j 1}^{(m)} & \mathcal{B}_{j 2}^{(m, n)} p_{j 1}^{(m)} & \ldots & \mathcal{B}_{j N}^{(m, n)} p_{j 1}^{(m)} \\
\mathcal{B}_{j 1}^{(m, n)} p_{j 2}^{(m)} & \mathcal{B}_{j 2}^{(m, n)} p_{j 2}^{(m)} & \ldots & \mathcal{B}_{j N}^{(m, n)} p_{j 2}^{(m)} \\
\vdots & & \ddots & \\
\mathcal{B}_{j 1}^{(m, n)} p_{j N}^{(m)} & \mathcal{B}_{j 2}^{(m, n)} p_{j N}^{(m)} & \ldots & \mathcal{B}_{j N}^{(m, n)} p_{j N}^{(m)}
\end{array}\right]
$$




\section{STATIONARY ANALYSIS}

In this section, we investigate the existence of a stationary solution of the recursion (1) in a stationary ergodic framework, and obtain expressions for the first two moments of this solution. For this, we make the following additional assumptions.

- The process $\left\{B_{n}, n \in \mathbb{Z}\right\}$ is stationary ergodic.

- The Markov chain $Y_{n}$ is ergodic.

- The processes $A_{n}$ are independent and identically distributed.

In view of these assumptions, we may simplify notation as follows: $\mathcal{A}_{i}^{(n)}=\mathcal{A}_{i}, b_{i}^{(n)}=b_{i}, \hat{b}^{(n)}=\hat{b}, \mathcal{B}_{i j}^{(m, n)}=\mathcal{B}_{i j}^{(n-m)}$, $p_{i j}^{(n)}=p_{i j}, P^{(n)}=P, \pi_{k}^{(n)}=\pi_{k}, \hat{\mathcal{A}}^{(n)}=\hat{\mathcal{A}}$ and $\hat{\mathcal{B}}^{(m, n)}=$ $\hat{\mathcal{B}}^{(n-m)}$. For ease of notation, the following operator will prove useful: for any $x \in \mathbb{R}^{M}$, let $\bigotimes_{i=n}^{k} A_{i}\left(x, Y_{i}\right)=x$ for $k<n$ whereas, for $k \geq n$, this operator is defined by the following recursion,

$$
\bigotimes_{i=n}^{k} A_{i}\left(x, Y_{i}\right)=A_{k}\left(\bigotimes_{i=n}^{k-1} A_{i}\left(x, Y_{i}\right), Y_{k}\right) .
$$

The operator above can be applied analogously on $\hat{A}_{i}^{(l)}$ for each $l$. We now state the stability theorem.

Theorem 1: Assume that (i) $b_{i}<\infty$ component-wise for all $i \in \Theta$; and (ii) that all the eigenvalues of the matrix $\hat{\mathcal{A}}$ are within the open unit disk. Then, there exist a unique stationary solution $X_{n}^{*}$, distributed like,

$$
X_{n}^{*}={ }_{d} \sum_{j=0}^{\infty} \bigotimes_{i=n-j}^{n-1} \hat{A}_{i}^{(n-j)}\left(B_{n-j-1}\left(Y_{n-j-1}\right), Y_{i}\right),
$$

for $n \in \mathbb{Z}$. The sum on the right side of the former expression converges absolutely almost surely. Furthermore, one can construct a probability space such that $\lim _{n \rightarrow \infty}\left\|X_{n}-X_{n}^{*}\right\|$ $=0$, almost surely, for any initial value $X_{0}$.

Proof: The proof follows a standard Loynes scheme. We define on the same probability space, the sequence of processes $X^{[l]}=\left\{X_{n}^{[l]} ; n \in \mathbb{Z}, n \geq-l\right\}$, with initial state zero $X_{-l}^{[l]}=0$ and governed by the recursive equation (1). One easily verifies that for fixed $n$, the sequence $X_{n}^{[l]}$ is monotone increasing (component-wise) in $l$. Hence, the limit $\lim _{l \rightarrow \infty} X_{n}^{[l]} \triangleq X_{n}^{*}$ is well defined; the right-hand side of (8) follows by consecutively applying the recursion (1), taking into account the divisibility (2).

Since, $X_{n}^{*}$ is measurable on the tail $\sigma$-algebra of the stationary ergodic sequence $\left\{\left(A_{n}\left(\cdot, Y_{n}\right), B_{n}\left(Y_{n}\right)\right) ; n \in \mathbb{Z}\right\}$, it is either finite, almost surely, or infinite, almost surely. In view of the recursion (1), we find,

$\mathrm{E}\left[X_{n+1}^{[l]} 1\left\{Y_{n+1}=j\right\}\right]=\sum_{i \in \Theta} \mathcal{A}_{i} \mathrm{E}\left[X_{n}^{[l]} 1\left\{Y_{n}=i\right\}\right] p_{i j}+b_{i} \pi_{i} p_{i j}$

for all $j \in \Theta$. Here 1\{\} is the indicator function which equals 1 if its argument is true and 0 if this is not the case. The former system of equations can be expressed in matrix notation as,

$$
\mu_{n+1}^{[l]}=\hat{\mathcal{A}} \mu_{n}^{[l]}+\hat{b} .
$$

$\mu_{n}^{[l]}$ denotes a (block) column vector with elements $\mu_{n}^{[l]}(j) \triangleq$ $\mathrm{E}\left[X_{n}^{[l]} 1\left\{Y_{n}=j\right\}\right](j \in \Theta)$. In view of the former expression, one easily verifies by induction that $\mu_{n}^{[l]} \leq(\mathcal{I}-\hat{\mathcal{A}})^{-1} \hat{b}$, component-wise. This then implies that $\mathrm{E}\left[X_{n}^{[l]}\right]$ is bounded above by a finite constant for all $l \geq 0, n \geq-l$ which in turn implies that $\mathrm{E}\left[X_{n}^{*}\right]$ is bounded above. Since $X_{n}^{*}$ is either finite, almost surely or infinite almost surely, we conclude that $X_{n}^{*}$ is finite, almost surely.

Consider now, the process $\left\{X_{n} ; n \in \mathbb{N}\right\}$, governed by the recursion (1), with initial state $X_{0}$. Consecutively applying the recursion yields,

$$
\begin{aligned}
X_{n}=\sum_{j=0}^{n-1}\left(\bigotimes_{i=n-j}^{n-1} \hat{A}_{i}^{(n-j)}\left(B_{n-j-1}\left(Y_{n-j-1}\right), Y_{i}\right)\right. & \\
& \left.+\bigotimes_{i=0}^{n-1} \hat{A}_{i}^{(0)}\left(X_{0}, Y_{i}\right)\right)
\end{aligned}
$$

for $n \in \mathbb{N}_{+}$. Hence, we have,

$$
X_{n}-X_{n}^{*}=\bigotimes_{i=0}^{n-1} \hat{A}_{i}^{(0)}\left(X_{0}, Y_{i}\right)-\bigotimes_{i=0}^{n-1} \hat{A}_{i}^{(0)}\left(X_{0}^{*}, Y_{i}\right),
$$

for $n \in \mathbb{N}_{+}$. We now show that both terms on the right-hand side of (9) converge to 0 almost surely.

Let $\hat{\mu}_{n}(x)$ denote the block column vector with elements,

$$
\mathrm{E}\left[\bigotimes_{i=0}^{n-1} \hat{A}_{i}^{(0)}\left(x, Y_{i}\right) 1\left\{Y_{n}=l\right\}\right], \quad l \in \Theta .
$$

By conditioning on the state of the Markov chain $Y$ and in view of the linearity in the mean (3), we find,

$$
\hat{\mu}_{n}(x)=\hat{\mathcal{A}} \hat{\mu}_{n-1}(x)=\hat{\mathcal{A}}^{n} \hat{\mu}_{0}(x), \quad n \in \mathbb{N}_{+},
$$

where $\hat{\mu}_{0}(x)$ denotes a block column vector whose $j$ th element equals $\pi_{j} x$. Since $\hat{\mathcal{A}}$ has no eigenvalues outside the open unit disk, we have $\lim _{n \rightarrow \infty} \hat{\mu}_{n}(x)=0$ for all $x \in \mathbb{R}^{M}$, which implies,

$$
\lim _{n \rightarrow \infty} \mathrm{E}\left[\bigotimes_{i=0}^{n-1} \hat{A}_{i}^{(0)}\left(x, Y_{i}\right)\right]=0 .
$$

Since, the sequence of random variables $\bigotimes_{i=0}^{n-1} \hat{A}_{i}^{(0)}\left(x, Y_{i}\right)$ is non-negative, application of Fatou's Lemma yields that this sequence converges to 0 almost surely. In view of (9), this shows that $X_{n}-X_{n}^{*}$ converges to 0 component-wise, which concludes the proof.

With the stability conditions established, we now focus on expressions for the first and second moments of $X_{0}^{*}$, conditioned on the state of the Markov chain $Y_{0}$. Let $\mu$, the conditional first moment vector, be the block column vector with elements $\mu_{i} \triangleq \mathrm{E}\left[X_{0}^{*} 1\left\{Y_{0}=i\right\}\right], i \in \Theta$. Analogously, let $\Omega$, the conditional second moment matrix, be the block column vector with elements $\Omega_{i} \triangleq \mathrm{E}\left[X_{0}^{*}\left(X_{0}^{*}\right)^{\prime} 1\left\{Y_{0}=i\right\}\right]$, $i \in \Theta$. The following theorem provides expressions for these vectors. 
Theorem 2: Assume that the stability conditions of Theorem 1 are satisfied. The conditional first moment vector is then given by,

$$
\mu=(\mathcal{I}-\hat{\mathcal{A}})^{-1} \hat{b} .
$$

Under the additional assumption that the second moments of $B_{0}(i)$ are finite, $i \in \Theta$, the elements $\Omega_{i}$ of the conditional second moment matrix of $X_{0}^{*}$ are the unique solution of the system of equations,

$$
\begin{aligned}
\Omega_{l}=\sum_{k \in \Theta}\left(F_{k}\left(\Omega_{k}\right)+\sum_{j=1}^{M} \mu_{k}^{(j)} \Gamma_{k}^{(j)}\right. & +\mathcal{B}_{k k}^{(0)} \pi_{k} \\
& \left.+\mathcal{A}_{k} \Lambda_{k}+\Lambda_{k}^{\prime} \mathcal{A}_{k}^{\prime}\right) p_{k l},
\end{aligned}
$$

$l \in \Theta$, where $\Lambda_{k}$ denotes the $k$ th diagonal (block) element of $\sum_{j=0}^{\infty} \hat{\mathcal{A}}^{j} \hat{\mathcal{B}}^{(j+1)}$ and with $\mu_{k}^{(j)}$ the $j$ th element of $\mu_{k}$.

Proof: Taking the expectations in (1) immediately yields the following system of equations,

$$
\begin{aligned}
\mu_{l} & =\mathrm{E}\left[X_{1}^{*} 1\left\{Y_{1}=l\right\}\right] \\
& =\sum_{k \in \Theta} \mathrm{E}\left[\left(A_{0}\left(X_{0}^{*}, k\right)+B_{0}(k)\right) 1\left\{Y_{0}=k, Y_{1}=l\right\}\right] \\
& =\sum_{k \in \Theta} \mathcal{A}_{k} \mu_{k} p_{k l}+\sum_{k \in \Theta} b_{k} p_{k l} \pi_{k}, \quad l \in \Theta .
\end{aligned}
$$

In block matrix notation, this system of equations is equivalent to,

$$
\mu=\hat{\mathcal{A}} \mu+\hat{b},
$$

which implies (10). Notice that $\mathcal{I}-\hat{\mathcal{A}}$ is non-singular since $\hat{\mathcal{A}}$ has only eigenvalues within the unit disk.

For the second moment, we first focus on the set of matrices $\Phi_{k l}(n)$,

$$
\Phi_{k l}(n)=\mathrm{E}\left[X_{0}^{*} B_{n}(l)^{\prime} 1\left\{Y_{0}=k\right\}\right], \quad k, l \in \Theta, n \in \mathbb{N} .
$$

In view of the recursion (1) this matrix satisfies,

$$
\begin{aligned}
\Phi_{k l}(n) & =\mathrm{E}\left[X_{1}^{*} B_{n+1}(l)^{\prime} 1\left\{Y_{1}=k\right\}\right] \\
& =\sum_{j \in \Theta} \mathrm{E}\left[\left(A_{0}\left(X_{0}^{*}, j\right)+B_{0}(j)\right) B_{n+1}(l)^{\prime} 1\left\{Y_{0}=j\right\}\right] p_{j k} \\
& =\sum_{j \in \Theta} \mathcal{A}_{j} \Phi_{j l}(n+1) p_{j k}+\mathcal{B}_{j l}^{(n+1)} \pi_{j} p_{j k},
\end{aligned}
$$

for $k, l \in \Theta, i \in \mathbb{N}$. In matrix notation, this system of equations reads,

$$
\Phi(n)=\hat{\mathcal{A}} \Phi(n+1)+\hat{\mathcal{B}}^{(n+1)}=\sum_{j=0}^{\infty} \hat{\mathcal{A}}^{j} \hat{\mathcal{B}}^{(n+j+1)},
$$

where $\Phi(i)$ denotes the block matrix with elements $\Phi_{k l}(i)$, $k, l \in \Theta$. Notice that the sum in (12) converges to a finitevalued matrix since (i) the finiteness of the second moments of $B_{0}(i)(i \in \Theta)$ implies that the elements of $\hat{\mathcal{B}}^{(n)}$ are uniformly bounded and since (ii) the eigenvalues of $\hat{A}$ are within the unit disk.
With the expression of $\Phi(i)$ at hand, and in view of the recursion (1), we immediately find,

$$
\begin{aligned}
& \Omega_{l}=\mathrm{E}\left[X_{1}^{*}\left(X_{1}^{*}\right)^{\prime} 1\left\{Y_{1}=l\right\}\right]=\sum_{k \in \Theta} \mathrm{E}\left[( A _ { 0 } ( X _ { 0 } ^ { * } , k ) + B _ { 0 } ( k ) ) \left(A_{0}\left(X_{0}^{*}, k\right)\right.\right. \\
&\left.\left.\quad+B_{0}(k)\right)^{\prime} 1\left\{Y_{0}=k, Y_{1}=l\right\}\right] \\
&=\sum_{k \in \Theta}\left(\begin{array}{c}
\left.F_{k}\left(\Omega_{k}\right)+\sum_{j=1}^{M} \mu_{k}^{(j)} \Gamma_{k}^{(j)}\right) \\
\quad p_{k l}+\sum_{k \in \Theta} \mathcal{B}_{k k}^{(0)} \pi_{k} p_{k l} \\
\quad+\sum_{k \in \Theta} \mathcal{A}_{k} \Phi_{k k}(0) p_{k l}+\sum_{k \in \Theta} \Phi_{k k}(0)^{\prime} \mathcal{A}_{k}^{\prime} p_{k l} .
\end{array}\right.
\end{aligned}
$$

Here $\mu_{i}^{(j)}$ denotes the $j$ th element of the vector $\mu_{i}$. This completes the proof.

\section{TRANSIENT ANALYSIS}

The previous section focused on the existence and the moments of the steady state process. We now consider its transient analysis. Here, no additional assumptions are required but the assumption that the initial vector $X_{0}$ and the initial Markov state $Y_{0}$ are independent from the processes $A_{n}$ and $B_{n}$.

Let $\mu(n)$ denote the conditional first moment vector at slot $n$. That is, $\mu(n)$ is the column vector whose $k$ th (block) element equals $\mu_{k}(n)=\mathrm{E}\left[X_{n} 1\left\{Y_{n}=l\right\}\right]$. Analogously, let $\Omega(n)$ denote the conditional second order moment (block) column vector whose $k$ th element equals $\Omega_{k}(n)=\mathrm{E}\left[X_{n} X_{n}^{\prime} 1\left\{Y_{n}=\right.\right.$ $l\}]$. Expressions for these block vectors are given in the Theorem below.

Theorem 3: The first moment vector at slot $n+1$ can be obtained recursively by,

$$
\mu(n+1)=\hat{\mathcal{A}}^{(n)} \mu(n)+\hat{b}^{(n)}
$$

The second moment vector at slot $n+1$ can be obtained by the following recursion

$$
\begin{aligned}
\Omega_{l}(n+1)= & \sum_{k \in \Theta}\left(F_{k}^{(n)}\left(\Omega_{k}(n)\right)+\sum_{j=1}^{M} \mu_{k}^{(j)}(n) \Gamma_{k, j}^{(n)}\right) p_{k l}^{(n)} \\
& +\sum_{k \in \Theta} \mathcal{B}_{k k}^{(n, n)} \pi_{k}^{(n)} p_{k l}^{(n)}+\sum_{k \in \Theta} \mathcal{A}_{k}^{(n)} \Phi_{k, k}^{(n, n)} p_{k l}^{(n)} \\
& +\sum_{k \in \Theta}\left(\Phi_{k, k}^{(n, n)}\right)^{\prime}\left(\mathcal{A}_{k}^{(n)}\right)^{\prime} p_{k l}^{(n)}
\end{aligned}
$$

with $\Phi_{k, k}^{(n, n)}$ the $k$ th diagonal (block) element of

$$
\begin{aligned}
\Phi^{(n, n)}=\hat{\mathcal{A}}^{(n-1)} \hat{\mathcal{A}}^{(n-2)} \cdots \hat{\mathcal{A}}^{(0)} \Phi^{(0, n)} & \\
& +\sum_{k=0}^{n-1} \hat{\mathcal{A}}^{(n-1)} \hat{\mathcal{A}}^{(n-2)} \cdots \hat{\mathcal{A}}^{(k+1)} \hat{\mathcal{B}}^{(k)},
\end{aligned}
$$

and where $\Phi^{(0, n)}$ is a block matrix with elements $\Phi_{k, l}^{(0, n)}=$ $\mu_{k}(0)\left(b_{l}^{(n)}\right)^{\prime}, k, l \in \Theta$.

Proof: Taking the expectations in (1) immediately yields the following system of equations,

$\mu_{l}(n+1)=\sum_{k \in \Theta} \mathcal{A}_{k}^{(n)} \mu_{k}(n) p_{k l}^{(n)}+\sum_{k \in \Theta} b_{k}^{(n)} p_{k l}^{(n)} \pi_{k}^{(n)}, \quad l \in \Theta$. 
which yields (13). Proceeding analogously, we find (14) for $\Omega_{i}(n)$,

$$
\begin{aligned}
\Omega_{l}(n+1)= & \mathrm{E}\left[X_{n+1}\left(X_{n+1}\right)^{\prime} 1\left\{Y_{n+1}=l\right\}\right] \\
= & \sum_{k \in \Theta}\left(F_{k}^{(n)}\left(\Omega_{k}(n)\right)+\sum_{j=1}^{M} \mu_{k}^{(j)}(n) \Gamma_{k, j}^{(n)}\right) p_{k l}^{(n)} \\
& +\sum_{k \in \Theta} \mathcal{B}_{k k}^{(n, n)} \pi_{k}^{(n)} p_{k l}^{(n)} \\
& +\sum_{k \in \Theta} \mathcal{A}_{k}^{(n)} \Phi_{k, k}^{(n, n)} p_{k l}^{(n)}+\sum_{k \in \Theta}\left(\Phi_{k, k}^{(n, n)}\right)^{\prime}\left(\mathcal{A}_{k}^{(n)}\right)^{\prime} p_{k l}^{(n)},
\end{aligned}
$$

with,

$$
\Phi_{k, l}^{(m, n)}=\mathrm{E}\left[X_{m} B_{n}(l)^{\prime} 1\left\{Y_{m}=k\right\}\right] .
$$

For the latter expectation, we further have the following recursion,

$$
\begin{aligned}
& \Phi_{k, l}^{(m, n)}=\sum_{j \in \Theta} \mathrm{E}\left[\left(A_{m-1}\left(X_{m-1}, j\right)+B_{m-1}(j)\right)\right.\left.B_{n}(l)^{\prime} 1\left\{Y_{m-1}=j, Y_{m}=k\right\}\right] \\
&=\sum_{j \in \Theta} \mathcal{A}_{j}^{(m-1)} \Phi_{j, l}^{(m-1, n)} p_{j k}^{(m-1)} \\
&+\mathcal{B}_{j, l}^{(m-1, n)} \pi_{j}^{(m-1)} p_{j k}^{(m-1)},
\end{aligned}
$$

whereas the independence of the initial state yields,

$$
\Phi_{k, l}^{(0, n)}=\mathrm{E}\left[X_{0} B_{n}(l)^{\prime} 1\left\{Y_{0}=k\right\}\right]=\mu_{k}(0)\left(b_{l}^{(n)}\right)^{\prime} .
$$

The system of equations (16), can be rewritten in block matrix notation,

$$
\Phi^{(m, n)}=\hat{\mathcal{A}}^{(m-1)} \Phi^{(m-1, n)}+\hat{\mathcal{B}}^{(m-1)}
$$

Solving the recursion then yields (15), which completes the proof.

\section{Applications in Delay Tolerant Mobile Ad-HoC NETWORKS}

With the theory established, we now focus on some applications in the context of packet-forwarding in delay tolerant mobile ad-hoc networks. The first two examples concern models with a fixed number of nodes. The last two examples are concerned with models where the number of nodes vary during time.

\section{A. Fixed number of mobile nodes}

As a first example, we consider a network that consists of $N$ mobile nodes. Some fixed node wishes to send a packet to a destination node. As connectivity is assumed to be low, the source makes use of the mobility of other mobiles that serve as relays. Whenever the source is within the transmission range of another node, it transmits a packet to that node. Whenever a node with a copy of a packet is within the transmission range of the destination then it transmits the packet to it.

Remark 1: In this example as well as in all the following ones, we restrict to the two-hop routing scheme [6]; we do not consider epidemic routing, i.e. a relay node that receives a packet from the source does not relay it further to other intermediate nodes.

Time is discrete and it is assumed that at each time $n$, each node has a probability $p_{i}^{(n)} \geq p>0$ to meet each other node; this probability also depends on the state $i \in \Theta$ of a modulating Markov chain. The transition matrix of this chain at time $n$ is denoted by $P^{(n)}=\left[p_{i j}^{(n)}\right]_{i, j \in \Theta}$.

The validity of (a continuous time version of) this model without the random environment has been discussed in [8], and its accuracy has been shown for a number of mobility models (Random Walker, Random Direction, Random Waypoint). The random environment enables us to further capture the fluctuations in time of the connectivity probability. For example, reduced connectivity probabilities may occur during rain storms which result in worse radio channel conditions.

Let $\xi_{n}(\ell, j)$ denote the indicator that equals 1 if nodes $\ell$ and $j$ meet at time $n$ and is otherwise 0 . Let $\left(\xi_{n}^{(i)}\right)_{n, i}$ be i.i.d. copies of $\xi_{n}$ Let $X_{n}$ be the number of nodes with a copy of the packet at time $n$, then the following recursion can be established,

$$
X_{n+1}=X_{n}+\sum_{i=1}^{N-X_{n}} \xi_{n}^{(i)} .
$$

The framework established in the preceding sections cannot be used directly for this recursion. A simple change of variables, $\tilde{X}_{n}=N-X_{N}$, however yields a recursion which is captured by our framework,

$$
\tilde{X}_{n+1}=\tilde{X}_{n}-\sum_{i=1}^{\tilde{X}_{n}} \xi_{n}^{(i)}=A_{n}\left(\tilde{X}_{n}\right)+B_{n} .
$$

where

$$
A_{n}\left(\tilde{X}_{n}\right)=\tilde{X}_{n}-\sum_{i=1}^{\tilde{X}_{n}} \xi_{n}^{(i)}, \quad B_{n}=0 .
$$

Notice that $\tilde{X}_{n}$ denotes the number of nodes that do not have a copy of the packet at time $n$. In view of the recursion for $\tilde{X}_{n}$, we obviously find $\hat{\mathcal{B}}^{(n)}=0$ and $\hat{b}^{(n)}=0$ for all $n$. Further, we have,

$$
\begin{aligned}
& \mathcal{A}_{i}^{(n)}=1-p_{i}^{(n)}, \quad F_{i}^{(n)}\left(x^{2}\right)=\left(1-p_{i}^{(n)}\right)^{2} x^{2}, \\
& \Gamma_{i, 1}^{(n)}=\left(1-p_{i}^{(n)}\right) p_{i}^{(n)}
\end{aligned}
$$

for $i \in \Theta$.

The steady state behaviour is trivial, $\tilde{X}_{n}$ converges to 0 and $X_{n}$ converges to $N$. Hence, we here focus on the transient behaviour. For this, we assume that the Markov chain is a timehomogeneous 2-state chain; state 1 (state 2) corresponding to low (high) interference levels. Let $\alpha$ and $\beta$ denote the transition probabilities from state 1 to state 2 and from state 2 to state 1 , respectively and let $p_{i}$ denote the probability that a node receives the packet in state $i(i=1,2)$. For ease of notation, let $\sigma=(1-\beta) /(2-\alpha-\beta)$ denote the (long-term) fraction of slots that the interference level is low (the chain is in state 1) and let the interference time 
$\tau=1 /(1-\alpha)+1 /(1-\beta)$ denote the mean length of a high and a low interference period.

In Figure 1, the time-evolution of the mean and the variance of the number of nodes that have the packet is depicted for different parameter sets; for all sets, we have $N=200$ nodes, $p_{1}=0.5 \%, p_{2}=10 \%$ and at time 0 the interference level is low. Further, following transition probabilities are chosen: $\alpha=$ $17 / 18$ and $\beta=1 / 2$ for set $1, \alpha=89 / 90$ and $\beta=9 / 10$ for set 2 and $\alpha=449 / 450$ and $\beta=49 / 50$ for set 3 . The fraction of time that the interference level is high is equal for all three sets. However, in comparison with set 1 , interference periods are on average 5 times (25 times) longer for set 2 (set 3 ). It is readily observed that the lengths of the interference periods have a huge impact on the performance. Longer periods yield a slower, more variable spreading of the packet among the nodes.

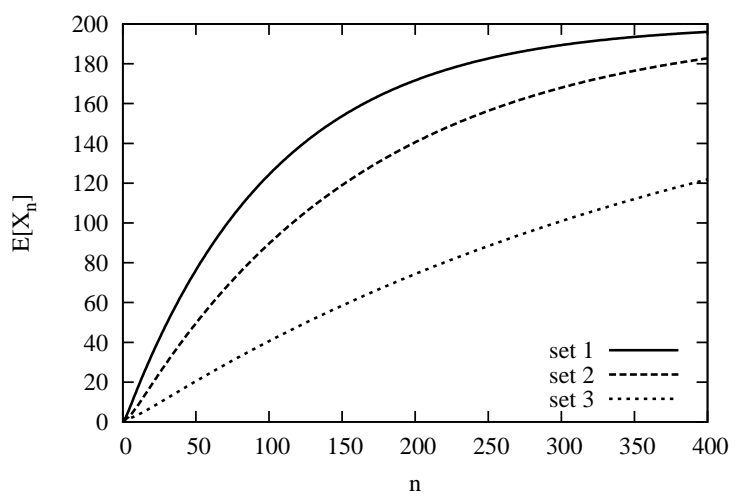

(a) mean

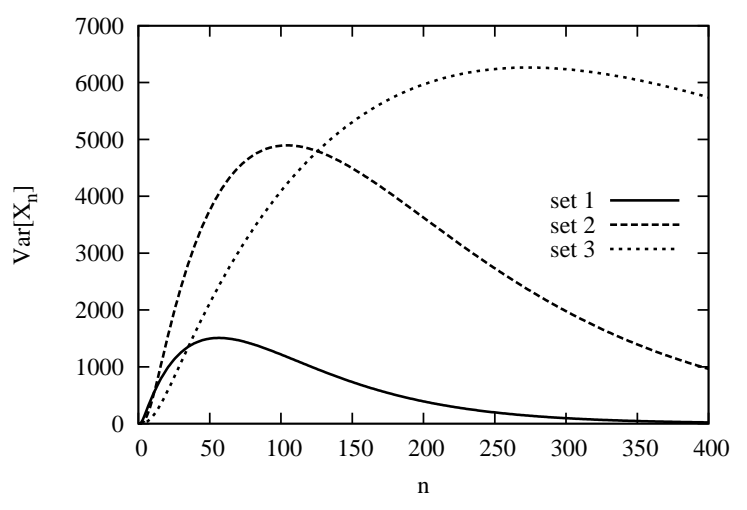

(b) variance

Fig. 1. Mean and variance of the number of nodes that have the packet as a function of time for various parameter settings.

\section{B. Packet discarding}

We now move to some models where the steady state behaviour is non-trivial.

In order to avoid packets to remain forever at nodes, it has been suggested to use expiration timers [15] for packets. Assume that each node uses a geometrically distributed initial value for the timer, with parameter $q$. The timer is initiated when a packet is received for relaying and the packet is discarded when the timer expires.

Retaining the assumptions and notation of the previous example, the number of nodes with the packet (excluding the source node) at consecutive slots are related as follows,

$$
X_{n+1}=\sum_{i=1}^{X_{n}} \zeta_{n}^{(i)}+\sum_{i=1}^{N-X_{n}} \xi_{n}^{(i)}
$$

Here, $\zeta_{n}^{(i)}$ are i.i.d. indicators that equal 0 if the $i$ th copy of a packet is discarded at time $n$. Note that for this model, the source node is not included in $X$ to ensure that the source does not discard the packet.

As in the previous example, this model is not directly covered by our framework. However, we may rewrite the equation as follows,

$$
\begin{aligned}
X_{n+1} & =\sum_{i=1}^{X_{n}} \zeta_{n}^{(i)}+\sum_{i=1}^{Y_{n}} \xi_{n}^{(i)} \\
Y_{n+1} & =N-\sum_{i=1}^{X_{n}} \zeta_{n}^{(i)}-\sum_{i=1}^{Y_{n}} \xi_{n}^{(i)} .
\end{aligned}
$$

In vector notation this set of equations can be written as follows,

$$
\left[\begin{array}{c}
X_{n+1} \\
Y_{n+1}
\end{array}\right]=A_{n}\left(\left[\begin{array}{c}
X_{n} \\
Y_{n}
\end{array}\right]\right)+B_{n}
$$

with,

$$
A_{n}\left(\left[\begin{array}{l}
x \\
y
\end{array}\right]\right)=\left(\sum_{i=1}^{x} \zeta_{n}^{(i)}+\sum_{i=1}^{y} \xi_{n}^{(i)}\right)\left[\begin{array}{c}
1 \\
-1
\end{array}\right], \quad B_{n}=\left[\begin{array}{c}
0 \\
N
\end{array}\right]
$$

This shows that our framework is applicable. We get the following matrices,

$$
\begin{aligned}
\mathcal{A}_{i}^{(n)} & =\left[\begin{array}{cc}
q & p_{i}^{(n)} \\
-q & -p_{i}^{(n)}
\end{array}\right], \\
\Gamma_{1, n}^{(i)} & =q(1-q)\left[\begin{array}{cc}
1 & -1 \\
-1 & 1
\end{array}\right], \\
\Gamma_{2, n}^{(i)} & =p_{i}^{(n)}\left(1-p_{i}^{(n)}\right)\left[\begin{array}{cc}
1 & -1 \\
-1 & 1
\end{array}\right],
\end{aligned}
$$

and,

$$
\begin{aligned}
F_{n}^{(i)}\left(\left[\begin{array}{ll}
x^{2} & x y \\
x y & y^{2}
\end{array}\right]\right) & = \\
& \left(x^{2} q^{2}+2 x y q p_{i}^{(n)}+y^{2}\left(p_{i}^{(n)}\right)^{2}\right)\left[\begin{array}{cc}
1 & -1 \\
-1 & 1
\end{array}\right] .
\end{aligned}
$$

Figure 2 depicts the mean and variance of the number of nodes that have the packet (in steady state) vs. the mean packet discarding time $T=1-1 / q$ for the same parameter sets as in Figure 1. Obviously, as nodes keep the packets longer, the mean number of nodes that have the packet increases. In contrast, the variance first increases with the discarding time and then decreases again. As in the previous example, correlation in the random environment negatively affects the mean number of nodes with the packet while the variance increases. 


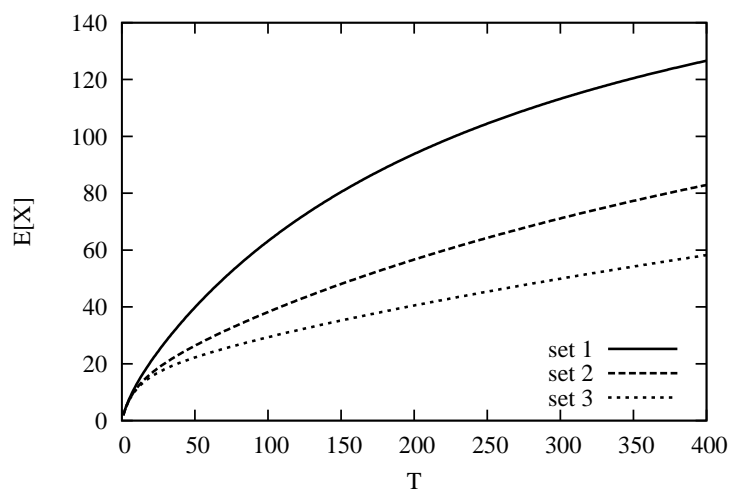

(a) mean

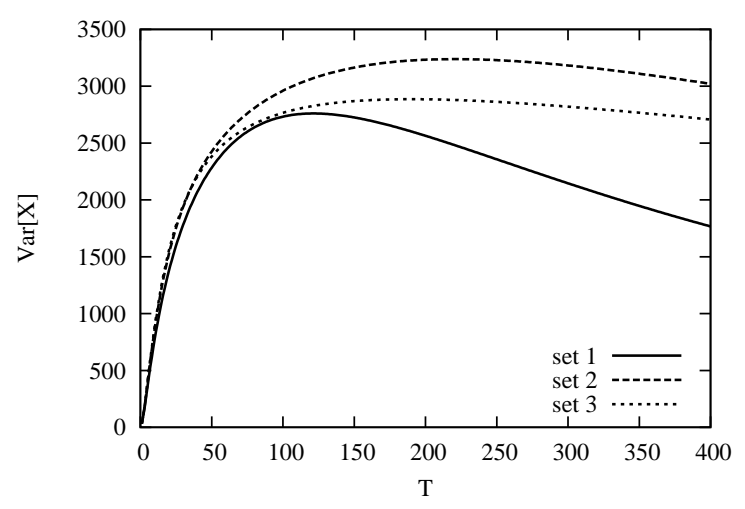

(b) variance

Fig. 2. Mean and variance of the number of nodes that have the packet as a function of the mean discarding time for various parameter settings.

\section{Variations in the total number of mobiles}

As before, some fixed source node wishes to send a packet to a destination node, thereby making use of the mobility of other mobiles that serve as relays. Whenever the source is within the transmission range of another node, it transmits the packet to that node. Whenever a node with a copy of the packet is within the transmission range of the destination, it transmits the packet to it. Further, we here adopt the two-state Markov environment of the previous examples, modelling the variations of the interference level.

In contrast to the previous examples, the number of nodes does not remain constant. New nodes arrive and nodes depart from the system. Let $W_{n}$ denote the number of nodes that have the packet at time slot $n$ and let $Z_{n}$ denote the number of nodes that do not have the file. We have the following recursion,

$$
\begin{aligned}
W_{n+1} & =\sum_{j=1}^{W_{n}} \zeta_{n, 1}^{(j)}+\sum_{j=1}^{Z_{n}} \zeta_{n, 2}^{(j)} \nu_{n}^{(j)} \\
Z_{n+1} & =\sum_{j=1}^{Z_{n}} \zeta_{n, 2}^{(j)}\left(1-\nu_{n}^{(j)}\right)+B_{n}
\end{aligned}
$$

Here $\zeta_{n, 1}^{(j)}$ is the indicator that the $j$ th node that has the packet leaves the system at slot $n, \zeta_{n, 2}^{(j)}$ is the indicator that the $j$ th node that does not have the packet leaves the system at slot $n$ and $\nu_{n}^{(j)}$ is the indicator that the $j$ th node that does not have the packet, receives the packet at slot $n$. Finally, $B_{n}$ denotes the number of new nodes that arrive during slot $n$.

We make the following assumptions. The indicators $\zeta_{n, 1}^{(j)}$ and $\zeta_{n, 1}^{(j)}$ constitute doubly indexed sequences of independent Bernoulli distributed random variables; let $q$ denote the probability that a node leaves the system. A node then remains in the system for $T=1 /(1-q)$ slots on average. Further, the indicators $\nu_{n}^{(i)}$ are Bernoulli distributed random variables whose distributions depend on the interference level (the state of the Markov chain) during slot $n$. Let $p_{1}\left(p_{2}\right)$ denote the probability that a node receives the packet if the Markov chain is in state 1 (state 2). Finally, the sequence of new nodes $B_{n}$ is stationary ergodic. Under these assumptions, the recursion (17) clearly fits the framework; let $X_{n} \triangleq\left[W_{k}, Z_{k}\right]^{\prime}$. Adhering to the notation of the framework of Sections II to IV, the following matrices and vectors characterise the recursion,

$$
\begin{aligned}
P & =\left[\begin{array}{cc}
\alpha & 1-\alpha \\
1-\beta & \beta
\end{array}\right], \quad \mathcal{A}_{i}=\left[\begin{array}{cc}
q & q p_{i} \\
0 & q\left(1-p_{i}\right)
\end{array}\right], \\
\Gamma_{1}^{(i)} & =\left[\begin{array}{cc}
q(1-q) & 0 \\
0 & 0
\end{array}\right], \\
\Gamma_{2}^{(i)} & =\left[\begin{array}{cc}
q p_{i}\left(1-q p_{i}\right) & -q^{2}\left(1-p_{i}\right) p_{i} \\
-q^{2}\left(1-p_{i}\right) p_{i} & q\left(1-p_{i}\right)\left(1-q\left(1-p_{i}\right)\right)
\end{array}\right],
\end{aligned}
$$

and,

$$
\begin{aligned}
F^{(i)}\left(x x^{\prime}\right)=x_{1}^{2} q^{2}\left[\begin{array}{ll}
1 & 0 \\
0 & 0
\end{array}\right] & +x_{1} x_{2} q^{2}\left[\begin{array}{cc}
2 p_{i} & 1-p_{i} \\
1-p_{i} & 0
\end{array}\right] \\
& +x_{2}^{2} q^{2}\left[\begin{array}{cc}
p_{i}^{2} & \left(1-p_{i}\right) p_{i} \\
\left(1-p_{i}\right) p_{i} & \left(1-p_{i}\right)^{2}
\end{array}\right],
\end{aligned}
$$

with $x=\left[x_{1}, x_{2}\right]^{\prime}$ and $i=1,2$.

We focus on the steady-state behaviour. Figure 3 depicts the mean number of nodes $\mathrm{E}[W]$ that have the packet and the mean number of nodes $\mathrm{E}[Z]$ that do not have the packet in steady-state. In Figure 3(a), these means are plotted vs. the mean residence time $T$ of the nodes for different values of the interference time $\tau$. The mean number of nodes in the system is equal to 50 ; the mean number of arrivals $\mathrm{E}[B]$ in a slot is reduced for increasing mean residence times. If there is no interference (state 1), a node receives the packet with probability $p_{1}=0.1$ whereas no transmission is possible during periods of high interference $\left(p_{2}=0\right)$. For all curves, the interference level is high during $\sigma=90 \%$ of the slots. It is readily observed that the mean residence time of a node has a considerable impact on $\mathrm{E}[W]$. Obviously, if nodes remain longer, they carry the packet for a longer time which explains the increase in the mean number of nodes that carry the packet. Further, increasing interference times yield decreasing $\mathrm{E}[W]$. This is confirmed by Figure $3(\mathrm{~b})$ where $\mathrm{E}[Z]$ and $\mathrm{E}[W]$ are depicted vs. the interference time $\tau$ for various values of the mean number of arrivals $\mathrm{E}[B]$. The same parameter setting is assumed as in Figure 3(a). 


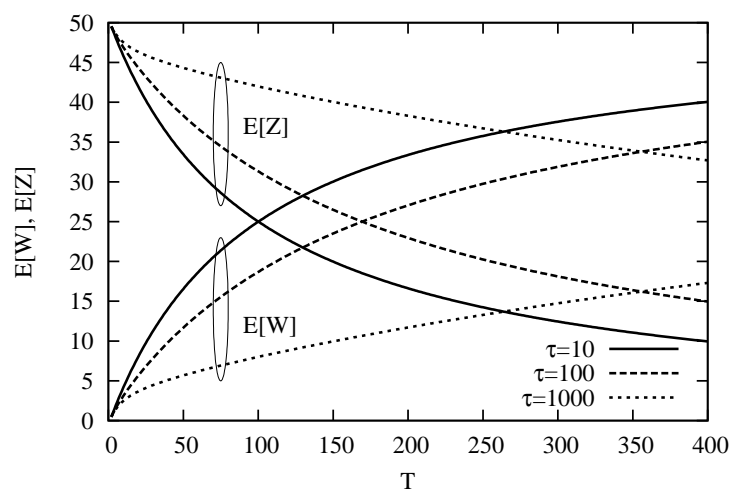

(a) mean vs. residence time

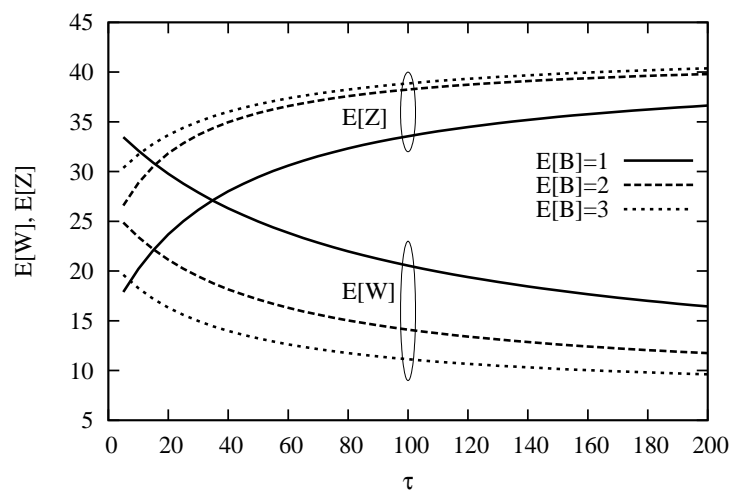

(b) mean vs. interference time

Fig. 3. Mean number of nodes that have the packet and of the number of nodes that do not have the packet vs. the mean residence time for various values of the mean interference time (a) and vs. the mean interference time for various values of the mean number of arrivals in a slot (b).

In accordance with equation (10), the mean value does not depend on the second order moments of the number of arrivals during the consecutive slots. The variance however does depend on these moments.

In Figures 4 and 5, the variance of the number of nodes with and without the packet are depicted versus the mean residence time (a) and versus the mean interference time (b) for the same parameters as in Figure 3. Additionally, the number of new arrivals are assumed to constitute a series of Poisson distributed random variables; this means that the variance of the number of new arrivals equals the mean number of new arrivals. These variables constitute an independent sequence in Figure 4 whereas their autocorrelation function $\rho(n)$ has a geometric decay $-\rho(n)=1 / 2^{n}-$ in Figure 5. Amongst others, discrete autoregressive processes of order 1 have such an autocorrelation function.

From these figures, it is readily observed that the variance of the number of nodes with (without) the packet is heavily affected by the mean residence time, the mean interference time and by the correlation of the number of new arrivals. For increasing values of the residence time, the variance of the number of nodes with (without) the packet first increases and then again decreases. A number of different effects are at play

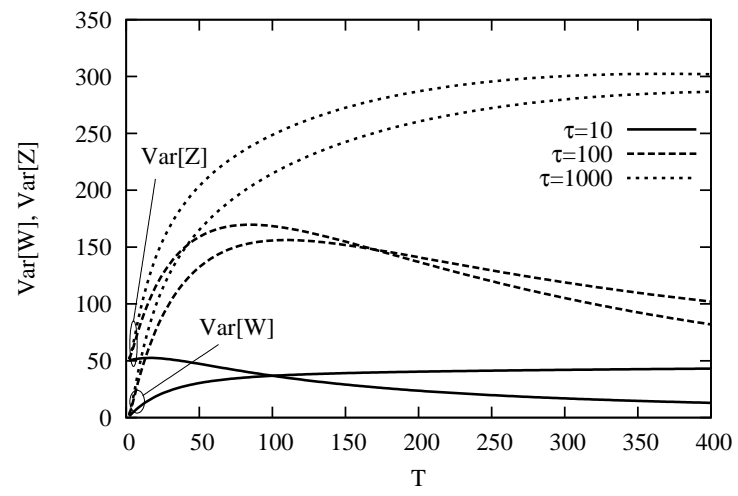

(a) variance vs. residence time

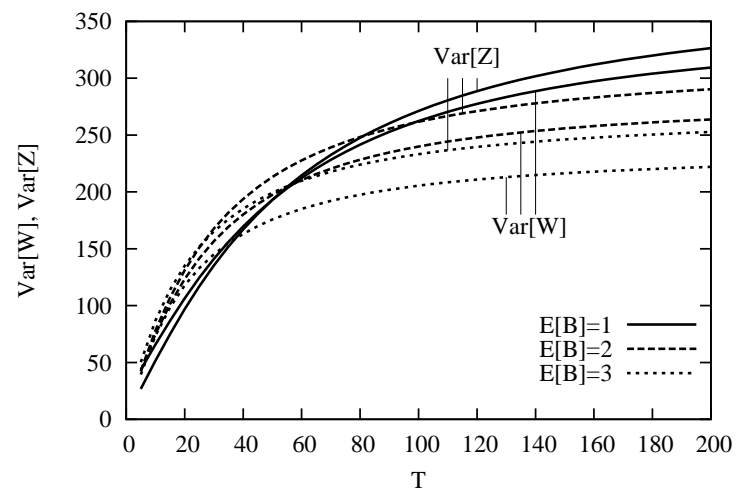

(b) variance vs. interference time

Fig. 4. Variance of the number of nodes that have the packet and of the number of nodes that do not have the packet vs. the mean residence time for various values of the mean interference time (a) and vs. the mean interference time for various values of the mean number of arrivals in a slot (b). There is no arrival correlation.

here. (i) Longer residence times imply that there are fewer arrivals which remain longer (recall that the mean number of nodes in the system is constant). As such, longer residence times may or may not increase the variance of the number of nodes in the system: on the one hand, the variance of the residence times increases, on the other hand the variance of the (Poisson-distributed) number of new arrivals decreases. (ii) Positive correlation in the arrival process increases the variance since arrivals are more clustered. (iii) Increasing the interference time means that there are longer periods with packet transmissions as well as longer periods without transmissions. Longer interference times thus imply higher variances.

\section{Mobility of the source and the nodes}

As a more advanced application of our framework, we now consider the following scenario. Again, some fixed source node wishes to send a packet to a destination node, thereby making use of the mobility of other mobiles that serve as relays. Whenever the source is within the transmission range of another node, it transmits the packet to that node. Whenever a node with a copy of a packet is within the transmission range of the destination, it transmits the packet to it. The source 


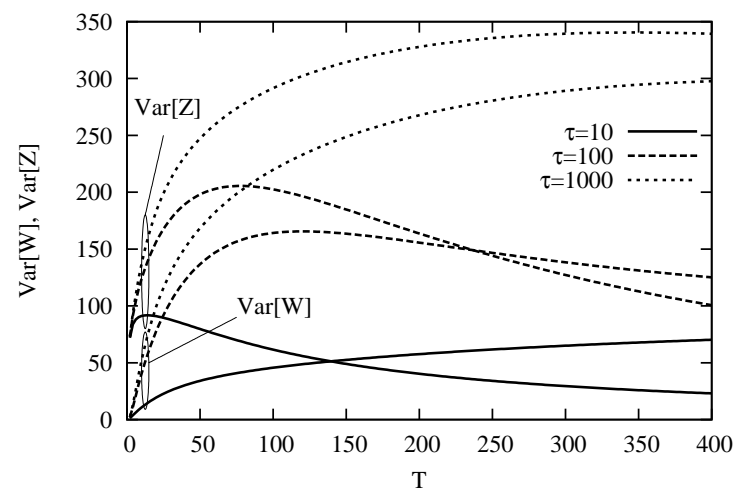

(a) mean vs. residence time

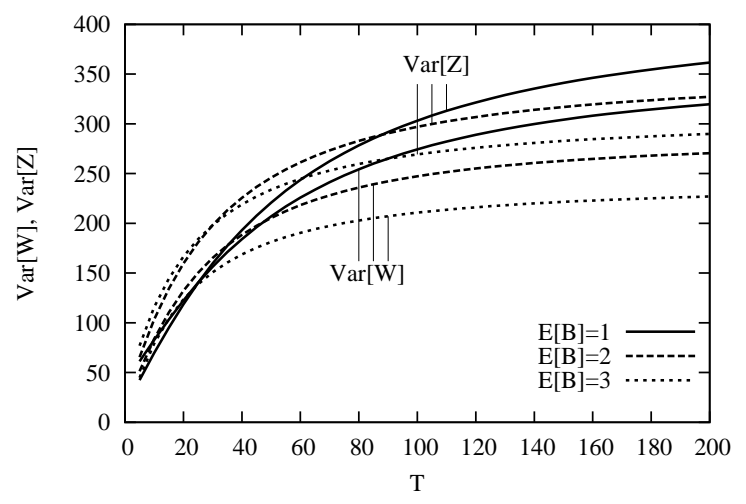

(b) mean vs. interference time

Fig. 5. Variance of the number of nodes that have the packet and of the number of nodes that do not have the packet vs. the mean residence time for various values of the mean interference time (a) and vs. the mean interference time for various values of the mean number of arrivals in a slot (b). The autocorrelation function of the new arrivals decays geometrically.

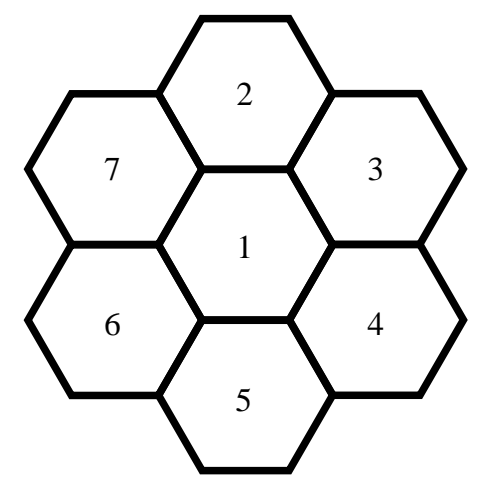

Fig. 6. Spatial grid of the nodes

node moves according to a random walk through a spatial grid, for example the spatial grid depicted in Figure 6. In each of the regions of the grid, new nodes arrive according to a stationary ergodic process which then travel through the grid until they leave. If a node is in the same region as the source, the node receives the packet with a fixed (possibly region-dependent) probability. In the remainder, let $N$ denote the number of regions.
Let $X_{n}(k)$ denote the number of nodes in region $k$ at time $n$ that have the packet and let $Z_{n}(k)$ denote the number of nodes that do not have the packet. Further let $X_{n}$ and $Z_{n}$ denote the column vectors with elements $X_{n}(k)$ and $Z_{n}(k)$, respectively. Let $Y_{n}$ denote the region where the source node resides at time $n$ and let $B_{n}(k)$ denote the number of new nodes that arrive in region $k$ at time $n ; B_{n}$ is the column vector with elements $B_{n}(k)$. We then have the following recursion,

$$
\begin{aligned}
X_{n+1} & =\sum_{i=1}^{N} \sum_{j=1}^{X_{n}(i)} \zeta_{n, 1}^{(i, j)}+\sum_{i=1}^{N} \sum_{j=1}^{Z_{n}(i)} \zeta_{n, 2}^{(i, j)} \nu_{n}^{(i, j)} \\
Z_{n+1} & =\sum_{i=1}^{N} \sum_{j=1}^{Z_{n}(i)} \zeta_{n, 2}^{(i, j)}\left(1-\nu_{n}^{(i, j)}\right)+B_{n}
\end{aligned}
$$

Here $\zeta_{n, 1}^{(i, j)}$ is a column vector of indicators; its $k$ th element is the indicator that the $j$ th node in region $i$ that has the packet at time $n$ moves to region $k$. The indicator vector $\zeta_{n, 2}^{(i, j)}$ is defined likewise. Its $k$ th element is the indicator that the $j$ th node in region $i$ that does not have the packet at time $n$ moves to region $k$. Further, $\nu_{n}^{(i, j)}$ denotes the indicator that the $j$ th node in region $i$ that does not have the packet at time $n$, receives the packet. Notice that some of the packets may leave the grid as not all packets necessarily move to any of the regions.

We make the following assumptions on these indicators. The indicators $\nu_{n}^{(i, j)}$ are Bernoulli distributed random variables whose distributions only depends on the position $Y_{n}$ of the source node. Further, a node moves from one region to another with a fixed probability. Hence, the vectors $\zeta_{n, 1}^{(i, j)}$ and $\zeta_{n, 1}^{(i, j)}$ constitute triply indexed sequences of independent and identically distributed random vectors. Finally, new nodes arrive according to a stationary ergodic process. We then have the following representation of recursion (18),

$$
\left[\begin{array}{c}
X_{n+1} \\
Z_{n+1}
\end{array}\right]=A_{n}\left(\left[\begin{array}{c}
X_{n} \\
Z_{n}
\end{array}\right], Y_{n}\right)+\left[\begin{array}{c}
0 \\
B_{n}
\end{array}\right]
$$

where the sequence $A_{n}$ is independent and identically distributed, adhering assumptions (2) to (4) such that our framework is applicable.

To limit the number of parameters involved, we make the following assumptions. All nodes move in the grid depicted in Figure 6. Each node remains in the same region during $T=1 /(1-\alpha)$ time intervals on average and then moves to any of the neighbouring regions with probability $1 / 6 . T$ is referred to as the mean residence time of the nodes. Analogously, the source node remains in the same region for $T$ slots on average and then moves to any of the neighbouring regions with equal probability. The source node never leaves the grid. A node that does not have the packet which is in the same region as the source node, receives the packet with probability $p$. Under these assumptions, the system parameters are determined in the appendix.

In Figure 7 the mean number of nodes with (a) and without (b) the packet in the different regions is depicted vs. the mean residence time $T$ in a region for different values of the 


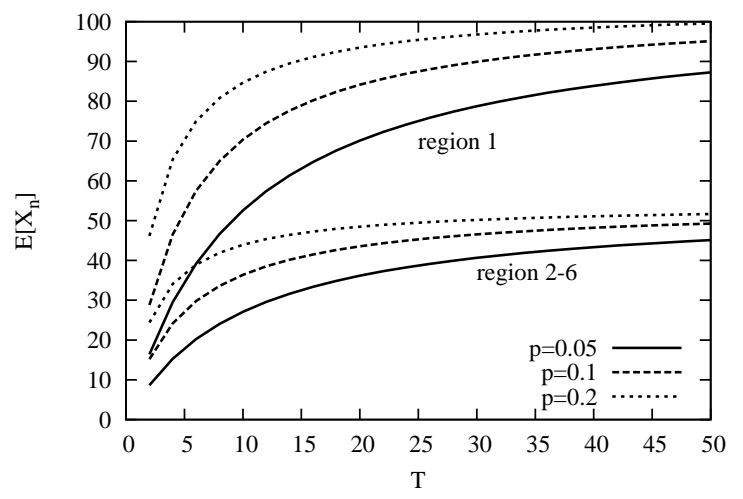

(a) Nodes with the packet

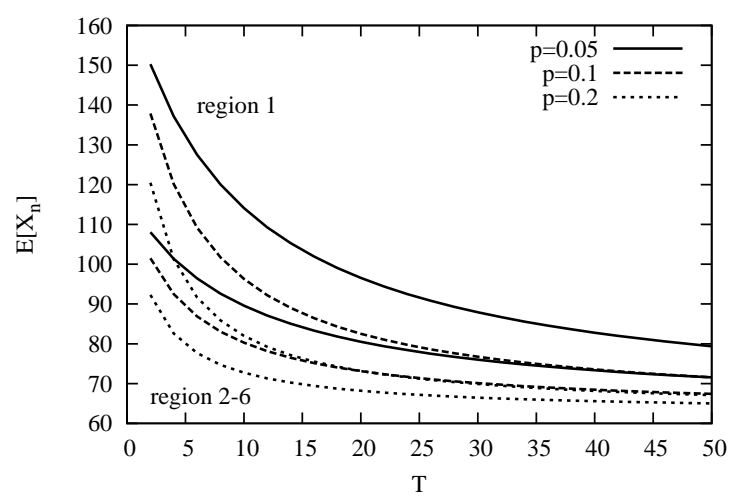

(b) Nodes without the packet

Fig. 7. Mean number of nodes with the packet (a) and without the packet (b) vs. the mean residence time in a region

transmission probability $p$. The number of new arrivals in the different regions scales with the residence times of the nodes: $\mathrm{E} B^{(i)}=50 / T$ for $i=1,2, \ldots, 7$ such that the total number of nodes in the different regions remains constant. First, notice that by symmetry, the characteristics of regions 2 to 7 are the same. Further, it is clear that longer residence times imply that more nodes receive the packet. Clearly, nodes do not only remain longer in a region but also longer in the grid. Hence, the probability that they receive the packet increases. Since the number of new arrivals is scaled with the residence times, the mean number of nodes without the packet decreases for increasing mean residence times; see Figure 7(b).

\section{CONCLUSiOns}

This paper provides expressions for the first two moments - both transient moments and steady state moments for stochastic recursive equations which encompasses both linear stochastic recursive equations and multi-type branching processes with immigration in a random environment. The immigration term in these recursions is taken to be stationary ergodic, whereas the random environment is taken to be Markovian. With the theory established, various examples in the context of delay-tolerant ad-hoc networks are developed: we find transient moments for a fixed number of mobile nodes in a Markovian environment and steady state moments for a fixed number of nodes with packet discarding after the exploration of an expiration timer, for a variable number of mobile nodes in a Markovian environment and for a variable number of mobile nodes moving in a grid.

\section{REFERENCES}

[1] E. Altman and D. Fiems. Expected waiting time in polling systems with correlated vacations. Queueing Systems, 56(3-4):241-253, 2007.

[2] K. Athreya and A. Vidyashankar. Handbook of Statistics, volume 19, chapter Branching Processes. Elsevier Science B.V., 2001.

[3] B. D'Auria. $M / M / \infty$ queues in semi-markovian random environment. Queueing Systems, 58(3):221-237, 2008.

[4] I. Eliazar. On the discrete-time $G / G I / \infty$ queue. Technical report, Holon Institute of Technology, 2006.

[5] G. Falin. The $M / M / \infty$ queue in a random environment. Queueing Systems, 58(1):65-76, 2008.

[6] M. Garetto, P. Giaccone, and E. Leonardi. On the effectiveness of the 2-hop routing strategy in mobile ad hoc networks. In Proceedings of the IEEE International Conference on Communications (ICC '07), 2007.

[7] S. A. Grishechkin. On a relation between processor sharing queues and Crump-Mode-Jagers branching processes. Advances in Applied Probability, 24:653-698, 1992.

[8] R. Groenevelt and P. Nain. Message delay in MANETs. In Proceedings of ACM SIGMETRICS, pages 412-413, Banff, June 2005.

[9] E. Key. Limiting distributions and regeneration times for multitype branching processes with immigration in a random environment. Annals of Probability, 15:344-353, 1987.

[10] I. MacPhee, M. Menshikov, D. Petritis, and S. Popov. A Markov chain model of a polling system with parameter regeneration. Annals of Applied Probability, 17(5-6):1447-1473, 2007.

[11] J. McNamara, A. Houston, and E. Collins. Optimality models in behavioral biology. SIAM Review, 43(3):413-466, 2001.

[12] R. Núñez Queija. Processor-Sharing Models for Integrated-Services Networks. PhD thesis, Eindhoven University of Technology, 2000.

[13] J. Resing. Polling systems and multi-type branching processes. Queueing Systems, 13:409-426, 1993.

[14] A. Roitershtein. A note on multitype branching processes with immigration in a random environment. Annals of Probability, 35(4):1573-1592, 2007.

[15] T. Small and Z. Haas. Resource and performance tradeoffs in delaytolerant wireless networks. In Proceedings of the ACM SIGCOMM workshop on Delay Tolerant Networks, Philadelphia, August 2005.

\section{APPENDIX}

In this appendix, we calculate the moments of the branching process of the mobility application of Section V-D. Under the assumptions of Section V-D, the transition matrix of the Markov chain $Y$ is given by the matrix $P$ below.

$$
P=\left[\begin{array}{ccccccc}
\alpha & \bar{\alpha} & \frac{\bar{\alpha}}{6} & \frac{\bar{\alpha}}{6} & \frac{\bar{\alpha}}{6} & \frac{\bar{\alpha}}{6} & \frac{\bar{\alpha}}{6} \\
\frac{\alpha}{3} & \alpha & \frac{\bar{\alpha}}{3} & 0 & 0 & 0 & \frac{\bar{\alpha}}{3} \\
\frac{\bar{\alpha}}{3} & \frac{\bar{\alpha}}{3} & \alpha & \frac{\bar{\alpha}}{3} & 0 & 0 & 0 \\
\frac{\bar{\alpha}}{3} & 0 & \frac{\bar{\alpha}}{3} & \alpha & \frac{\bar{\alpha}}{3} & 0 & 0 \\
\frac{\bar{\alpha}}{3} & 0 & 0 & \frac{\bar{\alpha}}{3} & \alpha & \frac{\bar{\alpha}}{3} & 0 \\
\frac{\bar{\alpha}}{3} & 0 & 0 & 0 & \bar{\alpha} & \alpha & \frac{\bar{\alpha}}{3} \\
\frac{\bar{\alpha}}{3} & \frac{\bar{\alpha}}{3} & 0 & 0 & 0 & \frac{\bar{\alpha}}{3} & \alpha
\end{array}\right]
$$

Further, we introduce the auxiliary sub-stochastic matrix $Q$, its entries being the probabilities that a node moves from one region to the other,

$$
Q=\left[\begin{array}{ccccccc}
\alpha & \overline{\frac{\alpha}{6}} & \frac{\bar{\alpha}}{6} & \frac{\bar{\alpha}}{6} & \frac{\bar{\alpha}}{6} & \frac{\bar{\alpha}}{6} & \frac{\bar{\alpha}}{6} \\
\frac{\bar{\alpha}}{6} & \alpha & \bar{\alpha} & 0 & 0 & 0 & \frac{\bar{\alpha}}{6} \\
\frac{\bar{\alpha}}{6} & \overline{\bar{\alpha}} & \alpha & \overline{\frac{\alpha}{6}} & 0 & 0 & 0 \\
\overline{\frac{\alpha}{6}} & 0 & \frac{\bar{\alpha}}{6} & \alpha & \frac{\bar{\alpha}}{6} & 0 & 0 \\
\frac{\bar{\alpha}}{6} & 0 & 0 & \overline{\frac{\alpha}{6}} & \alpha & \frac{\bar{\alpha}}{6} & 0 \\
\frac{\bar{\alpha}}{6} & 0 & 0 & 0 & \frac{\bar{\alpha}}{6} & \alpha & \frac{\bar{\alpha}}{6} \\
\frac{\bar{\alpha}}{6} & \overline{\frac{\alpha}{6}} & 0 & 0 & 0 & \frac{\bar{\alpha}}{6} & \alpha
\end{array}\right]
$$


Given the matrix $Q$ above, it is easily shown that the $14 \times$ 14 matrices $\hat{\mathcal{A}}_{k}$ which correspond with the process $A_{n}()$ of equation (19), are given by,

$$
\mathcal{A}_{k}=\left[\begin{array}{cc}
Q & Q S_{k} \\
0 & Q\left(I-S_{k}\right)
\end{array}\right]
$$

where $S_{k}$ denotes a $7 \times 7$ matrix of zeroes except for its $k$ diagonal element which equals $p$.

For the second moment, it is necessary to determine the linear operators $F^{(i)}$ and the matrices $\Gamma_{i, j}, i=1, \ldots, 7$, $j=1, \ldots, 14$. These immediately follow from the following expressions for the second order (cross) moments,

$$
\begin{aligned}
\mathrm{E} & {\left[Z_{n+1} Z_{n+1}^{\prime} \mid Z_{n}, Y_{n}=k, B_{n}=0\right] } \\
= & \sum_{i=1}^{N} \sum_{j=1}^{N} Z_{n}(i) Z_{n}(j) \mathrm{E}\left[\zeta_{n, 2}^{(i, 1)}\right] \mathrm{E}\left[\zeta_{n, 2}^{(j, 1)}\right]^{\prime} \\
& +\sum_{i=1}^{N} Z_{n}(i)\left(\mathrm{E}\left[\zeta_{n, 2}^{(i, 1)}\left(\zeta_{n, 2}^{(i, 1)}\right)^{\prime}\right]-\mathrm{E}\left[\zeta_{n, 2}^{(i, 1)}\right] \mathrm{E}\left[\zeta_{n, 2}^{(i, 1)}\right]^{\prime}\right) \\
& -p \sum_{i=1}^{N} Z_{n}(k) Z_{n}(i)\left(\mathrm{E}\left[\zeta_{n, 2}^{(k, 1)}\right] \mathrm{E}\left[\zeta_{n, 2}^{(i, 1)}\right]^{\prime}+\mathrm{E}\left[\zeta_{n, 2}^{(i, 1)}\right] \mathrm{E}\left[\zeta_{n, 2}^{(k, 1)}\right]^{\prime}\right) \\
& +p(2-p) Z_{n}(k) \mathrm{E}\left[\zeta_{n, 2}^{(k, 1)}\right] \mathrm{E}\left[\zeta_{n, 2}^{(k, 1)}\right]^{\prime} \\
& -p Z_{n}(k) E\left[\zeta_{n, 2}^{(k, 1)}\left(\zeta_{n, 2}^{(k, 1)}\right)^{\prime}\right]+p^{2} Z_{n}(k)^{2} \mathrm{E}\left[\zeta_{n, 2}^{(k, 1)}\right] \mathrm{E}\left[\zeta_{n, 2}^{(k, 1)}\right]^{\prime}, \\
\mathrm{E} & {\left[X_{n+1} X_{n+1}^{\prime} \mid X_{n}, Z_{n}, Y_{n}=k\right] } \\
= & \sum_{i=1}^{N} \sum_{j=1}^{N} X_{n}(i) X_{n}(j) \mathrm{E}\left[\zeta_{n, 1}^{(i, 1)}\right] \mathrm{E}\left[\zeta_{n, 1}^{(j, 1)}\right]^{\prime} \\
& \left.+\sum_{i=1}^{N} X_{n}(i)\left(\mathrm{E}\left[\zeta_{n, 1}^{(i, 1)}\left(\zeta_{n, 1}^{(i, 1)}\right)^{\prime}\right]-\mathrm{E}\left[\zeta_{n, 1}^{(i, 1)}\right] \mathrm{E}\left[\zeta_{n, 1}^{(i, 1)}\right]\right]^{\prime}\right) \\
& +p^{2} Z_{n}(k)^{2} \mathrm{E}\left[\zeta_{n, 2}^{(k, 1)}\right] \mathrm{E}\left[\zeta_{n, 2}^{(k, 1)}\right]^{\prime}+p(1-p) Z_{n}(k) \mathrm{E}\left[\zeta_{n, 2}^{(k, 1)}\left(\zeta_{n, 2}^{(k, 1)}\right)^{\prime}\right] \\
& +p \sum_{i=1}^{N} X_{n}(i) Z_{n}(k)\left(\mathrm{E}\left[\zeta_{n, 1}^{(i, 1)}\right] \mathrm{E}\left[\zeta_{n, 2}^{(k, 1)}\right]^{\prime}+\mathrm{E}\left[\zeta_{n, 2}^{(k, 1)}\right] \mathrm{E}\left[\zeta_{n, 1}^{(i, 1)}\right]^{\prime}\right) \\
& -p \\
& {\left[X_{n+1} Z_{n+1}^{\prime} \mid X_{n}, Z_{n}, Y_{n}=k, B_{n}=0\right] } \\
= & \sum_{i=1}^{N} \sum_{j=1}^{N} X_{n}(i) Z_{n}(j) \mathrm{E}\left[\zeta_{n, 1}^{(i, 1)}\right] \mathrm{E}\left[\zeta_{n, 2}^{(j, 1)}\right]_{n}^{\prime}(i) Z_{n}(k) \mathrm{E}\left[\zeta_{n, 1}^{(i, 1)}\right] \mathrm{E}\left[\zeta_{n, 2}^{(k, 1)}\right]^{\prime} \\
& \\
&
\end{aligned}
$$

